\title{
The economic costs of diabetes in Iran: some concerns and recommendations. Reply to Khuwaja AK, Khowaja LA, Cosgrove P [letter]
}

\author{
A. Esteghamati • O. Khalilzadeh • M. Abbasi
}

Received: 20 October 2009 / Accepted: 23 October 2009 /Published online: 18 November 2009

(C) Springer-Verlag 2009

Keywords Cost of illness · Diabetes · Direct cost $\cdot$ Indirect cost · Iran

To the Editor: There is currently great concern about the economic aspects of healthcare, including those related to diabetes [1]. We recently reported an estimation of the economic costs of diabetes in Iran [2]. Dr Khuwaja et al. have commented on our article [3] and highlighted their concern that we have underestimated these costs. We agree with their comment on the growing and disproportionate burden of diabetes in developing countries with a rather younger onset of the disease than elsewhere [4, 5]. In our original article [2] we noted that the costs related to diabetes are disproportionately high in Iran. We believe that in order to enable management of these costs and prevention of their escalation, there needs to be a thorough investigation of the size of the problem; there is also a need for design of national action plans and multidisciplinary approaches for the prevention, early diagnosis and management of diabetes and its complications. Nonetheless, we cannot accept the claim of Khuwaja et al. that our methods are imprecise and misleading. Here we provide clarification on this issue.

A. Esteghamati $(\square) \cdot$ O. Khalilzadeh $\cdot$ M. Abbasi Endocrinology and Metabolism Research Centre (EMRC),

Vali-Asr Hospital, School of Medicine,

Tehran University of Medical Sciences,

Tehran, Iran

e-mail: esteghamati@tums.ac.ir

M. Abbasi

Nuclear Medicine Research Centre, Shariati Hospital,

Tehran University of Medical Sciences,

Tehran, Iran
First, to determine the healthcare cost attributable to diabetes, a common approach is to compare the patterns of healthcare use by individuals with and without diabetes [6]. The prevalence rates of hypertension and dyslipidaemiaconsidered to be partially unrelated to diabetes - are equal or greater in the population with diabetes than in the population without diabetes. If we exclude participants with diseases other than diabetes, we would overestimate the excess costs of diabetes. Following the suggestion of Khuwaja et al., one would calculate an approximation of the overall health costs of diabetic patients instead of the attributable excess costs of diabetes. Our method is consistent with the methods used in other studies [6, 7]. Second, according to our Methods, 'Indirect costs were estimated in workers only.' Where possible, indirect costs should cover lost work hours both for those in and those not in the labour force, including housewives. In this way we did underestimate the diabetes-related expenditure. However, we reject the idea that we misled the reader, as we clearly defined our limited approach in the section headed 'Costing methods'. In the USA, the estimated value of lost housekeeping services for individuals not in the labour force is $20 \%$ of annual earnings for individuals in the labour force. However, there is no similar estimation available or verified for Iran. In addition, we did not record the costs related to restricted activity of the labour force, premature mortality (lost lifetime earnings) and permanent disability. Third, the inference of Khuwaja et al. that we included out-of-pocket expenses as indirect costs is not correct. In the Abstract and Methods we defined direct costs as including out-of-pocket expenditure. Fourth, the government of Iran subsidises healthcare through insurance and through direct subsidies, mainly for medications. The subsidies for the insurance system are included in the 
insurance share of the expenditures, and so were included in the calculations. Since 2002 the subsidies have been devoted to imported medicines, such as blood factors and some of the anti-cancer medicines, and not to locally manufactured medications, according to Cheraghali [8]. We estimate that about $80 \%$ of the direct subsidies are assigned for medications for a few illnesses (including cancers, haemophilia and multiple sclerosis) and the remaining $20 \%$ covers diabetes and other diseases. Thus, bearing in mind that the sample in our study was representative of the population of Tehran, we have not underestimated the diabetes-attributed costs - the difference in the costs for those with and without diabetes. Finally, inclusion of work hours lost by patients' attendants in the indirect costs of diseases is not commonly used $[6,7]$.

We believe that the recommendations of Khuwaja et al. [3] regarding the precision of the estimation of productivity loss of the patients and their attendants, and their comment on the complexity of the subsidies in systems similar to that in Iran, should be considered in the design of future studies.

Duality of interest The authors declare that there is no duality of interest associated with this manuscript.

\section{References}

1. Klonoff DC, Schwartz DM (2000) An economic analysis of interventions for diabetes. Diabetes Care 23:390-404

2. Esteghamati A, Khalilzadeh O, Anvari M et al (2009) The economic costs of diabetes: a population-based study in Tehran, Iran. Diabetologia 52:1520-1527

3. Khuwaja AK, Khowaja LA, Cosgrove P (2009) The economic costs of diabetes in developing countries: some concerns and recommendations. Diabetologia. doi:10.1007/s00125-009-1581-7

4. Esteghamati A, Gouya MM, Abbasi M et al (2008) Prevalence of diabetes and impaired fasting glucose in the adult population of Iran: national survey of risk factors for non-communicable diseases of Iran. Diabetes Care 31:96-98

5. Esteghamati A, Meysamie A, Khalilzadeh O et al (2009) Third national surveillance of risk factors of non-communicable diseases (SuRFNCD-2007) in Iran: methods and results on prevalence of diabetes, hypertension, obesity, central obesity, and dyslipidemia. BMC Public Health 9:167

6. Hogan P, Dall T, Nikolov P, American Diabetes Association (2003) Economic costs of diabetes in the US in 2002. Diabetes Care 26:917-932

7. American Diabetes Association (2008) Economic costs of diabetes in the U.S. in 2007. Diabetes Care 31:596-615 Erratum in Diabetes Care 2008: 31; 1271

8. Cheraghali AM (2006) Iran pharmaceutical market. Iran J Pharm Res $1: 1-7$ 